\title{
Uniform Price Mechanisms for Threshold Public Goods Provision with Complete Information: An Experimental Investigation
}

\begin{abstract}
We introduce two novel mechanisms for provision point public goods, motivated by the design of uniform price auctions: The uniform price auction mechanism (UPA) collects an endogenously determined uniform price from everyone offering at least that price, while the uniform price cap mechanism (UPC) collects the uniform price from everyone offering at least that price, plus the full offer of everyone offering less. UPC has the same undominated perfect equilibria as standard provision point (PPM) and proportional rebate (PR) mechanisms, and UPA a somewhat broader set. However, our mechanisms' different marginal penalty structures may facilitate equilibrium selection and lead to higher contributions and more frequent provision. Through laboratory experiments, using both homogeneous (symmetric) and heterogeneous induced values, we show our mechanisms improve upon PR and PPM: UPC generates higher aggregate contributions than PR and PPM, leading to higher provision rates than PPM; UPA attracts much higher contributions, although it provides less frequently. This ranking emerges because high offers are more common (especially among high value people in the heterogeneous environment) in the uniform price mechanisms, where higher offers only increase the payment when needed for provision.
\end{abstract}

Keywords: Uniform price auction, Uniform price cap, Proportional rebate, Provision point mechanism 


\section{Introduction}

A provision point public good is one that can be provided only when a threshold level of funding contributions is met. Canonical examples include bridges, parks and schools that require a discrete level of funding to cover building costs, but potentially continuous public goods such as public radio broadcasting and environmental conservation projects are sometimes marketed as provision point public goods in order to increase contributions. The public goods literature typically envisions determining outcomes through the provision point mechanism (PPM), in which people voluntarily and simultaneously contribute toward funding the good: if the total contribution reaches or exceeds the provision point (the threshold cost), the good is provided; otherwise contributions are refunded (money back guarantee). Because it is simpler than other public goods mechanisms in which provision is supported as a Nash equilibrium - in contrast to the unique zero-contribution prediction of the voluntary contribution mechanism-PPM has been widely studied, both theoretically ${ }^{1}$ and experimentally. ${ }^{2}$

However, PPM has a continuum of efficient Nash equilibria in which total contributions exactly equal the provision point (Bagnoli and Lipman, 1989), and theory is silent about the cost sharing rule. This equilibrium selection problem has led to an additional literature on whether and how different methods for rebating contributions in excess of the provision costattributable to imperfect coordination - affect the incentives for making contributions. Marks and Croson (1998) compare no-rebate, proportional rebate (PR), and a "utilization" rebate in the form of higher public good levels, and find a utilization rebate leads to higher contributions, but no significant difference between no-rebate and PR under complete information. When value information is private, Gailmard and Palfrey (2005) find PR (called PCS in their paper) generates significantly higher contributions than no-rebate. Only a few of the possible factors affecting these mixed results have been explored. Rondeau et al. (1999) identify a significant group size effect, but no information (about group size or provision point) effects in PR; Rondeau et al. (2005) find PR generates higher contributions compared with the voluntary contribution mechanism. Spencer et al. (2009) show PR and five other lottery-based rebate rules result in full demand revelation with a group size of 45 . These studies use one-shot games, and do not provide

\footnotetext{
${ }^{1}$ Bagnoli and Lipman (1989) study PPM under complete information; Nitzan and Romano (1990), McBride (2006), and Barbieri and Malueg (2010a) discuss threshold uncertainty; Alboth et al. (2001), Menezes et al. (2001), Laussel and Palfrey (2003), and Barbieri and Malueg (2008, 2010b) discuss PPM with private value information.

${ }^{2}$ See Chen (2008) for a recent review of related experimental studies; for earlier reviews see Davis and Holt (1993) and Ledyard (1995).
} 
comparisons between rebate mechanisms and a PPM without rebate. This study advances this literature by presenting a coherent framework for understanding how rebate rules affect contributions by differently "penalizing" higher offers when the provision point is exceeded, thus increasing or decreasing the chance of provision amid coordination failure, and uses that to develop two novel mechanisms that outperform PPM and PR.

Our novel mechanisms are motivated by payment rules in multi-unit uniform price auctions (cf. Gailmard and Palfrey (2005) on excludable public goods). In our uniform price auction mechanism (UPA), everyone who pays, pays the same price: if there exists a price such that the number of contributions at or above that price multiplied by the price equals the provision point, then the good is provided, with only those offering at or above the uniform price paying the uniform price; the lowest such price will be chosen if more than one uniform price is possible. Our second mechanism addresses the inefficiency inherent in UPA, that contributions can exceed the provision cost, but still no uniform price meeting the provision rule exists. In the uniform price cap mechanism (UPC), no one pays more than the uniform price: if the provision point is exceeded, the lowest price cap will be calculated so whoever contributes above the cap pays only the cap, and those contributing less than the cap pay their full offer, such that the final collected payments sum to the provision point.

The rebate rules in UPA and UPC present different incentives for making higher contributions than those of PPM and PR. Marks and Croson (1998) use the notion of "marginal penalty" to characterize differences among rebate rules, which they define as the cost to the contributor of contributing an additional dollar, conditioned on provision. For example, PPM has a marginal penalty of -1 since there is no rebate and none of the extra dollar will be returned, and the marginal penalty in PR is, in general, between -1 and 0. Marks and Croson (1998) argue that, when agents are unsure about others' contributions, because the expected costs of making higher offers is less when the marginal penalty is lower, mechanisms with lower marginal penalties will generate higher contributions and increase the likelihood of provision. Gailmard and Palfrey (2005) find this to be true with incomplete information, but the lack of effect with complete information (Marks and Croson, 1998) suggests that the role of marginal penalty is more nuanced. Our uniform price mechanisms provide a stronger test for the role of marginal penalty, because they introduce significant ranges of others' contributions where the marginal penalty is zero. If regions of zero marginal penalty lead agents to think it may cost them nothing to venture 
a higher contribution, UPA and UPC may improve performance over PPM and PR. This effect may be enhanced in the presence of other-regarding preferences as, relative to PPM and PR, those who make higher offers are not penalized with higher payments that lead to more unequal outcomes, unless the money is needed to secure provision.

Our experimental results show that, while average contribution behavior is consistent with the undominated perfect equilibrium predictions for each mechanism, the stable total contribution level near the provision cost (or above in the case of UPA) is the result of an ongoing coordination process wherein individual subjects continuously adjust their offers in an effort to cheap ride. ${ }^{3}$ Thus, provision rates are ultimately determined by the willingness of other subjects to offer more than their "fair share" of the cost. The aggregate willingness to make these higher contributions is affected by the risk of venturing a higher offer, as captured by the marginal penalty. We find UPC generates higher contributions and provision rates than PR and PPM, and UPA generates much higher contributions.

The rest of the paper is organized as follows. Section 2 defines precisely the four mechanisms to be compared. Section 3 characterizes the mechanisms' undominated perfect Nash equilibria, and their respective marginal penalty structures. Section 4 describes the experimental design and procedures. Section 5 discusses the observed group and individual contributions. Section 6 synthesizes the results.

\section{The Mechanisms}

Suppose $N$ agents each have endowment $I$. Each simultaneously chooses to make a contribution $c_{i} \in S_{i}=[0, I]$ to the provision of a threshold public good $g \in\{0,1\}$ with a cost function $T(g=1)$ $=P P, T(g=0)=0$. If the public good is provided, each agent receives a private value of $v_{\mathrm{i}}>0$. If the public good is not provided, all contributions are refunded (money-back guarantee). The group size $N$, the cost $P P$, and the value $v_{\mathrm{i}}$ of each group member are all common knowledge. Agent $i$ 's utility or payoff depends on the mechanism used, as described below.

\subsection{Provision Point Mechanism (PPM)}

The payoff function for agent $i$ under PPM is

$$
\text { (1) } \pi_{i}=\left\{\begin{array}{lc}
I-c_{i}+v_{i} & \text { if } \sum_{j=1}^{N} c_{j} \geq P P \\
I & \text { otherwise }
\end{array}\right.
$$

\footnotetext{
${ }^{3}$ Issac et al. (1989) used the notion of "cheap ride" to describe "individual incentives to attempt to obtain an equilibrium outcome with unequal distribution of contributions."
} 
Under PPM, if $P P$ is met or exceeded, each agent receives the initial endowment minus their contribution, plus their private value, $v_{\mathrm{i}}$, for the public good; otherwise, they only get the initial endowment.

If total contributions exceed $P P$, PPM "burns" the excess contributions. Alternatively, excess contributions can be returned through rebate mechanisms, which may affect contribution strategies. PR, UPA, and UPC return excess contributions in different ways.

\subsection{Proportional Rebate (PR)}

Agent $i$ 's payoff under PR is

(2) $\pi_{i}= \begin{cases}I-c_{i}+v_{i}+\frac{c_{i}}{\sum_{j=1}^{N} c_{j}}\left(\sum_{j=1}^{N} c_{j}-P P\right) & \text { if } \sum_{j=1}^{N} c_{j} \geq P P \\ I & \text { otherwise }\end{cases}$

Under PR, if $\sum_{j} c_{j}>P P$, the excess contribution $\left(\sum_{j} c_{j}-P P\right)$ will be rebated. The rebate to each agent is proportional to the ratio of their individual contribution to the total contribution. Suppose $P P=12, N=3$, then a contribution profile $c=\{11,8,6\}$ with a sum of 25 has 13 units of excess contribution and will result in a rebate profile of $\left\{\frac{13}{25} \cdot 11, \frac{13}{25} \cdot 8, \frac{13}{25} \cdot 6\right\}=\{5.72,4.16,3.12\}$.

\subsection{Uniform Price Auction (UPA)}

Under UPA, a uniform price, $U P$, will be calculated. $U P$ is the lowest price such that the number of contributions higher than that price times the price is equal to the provision point. The payoff under UPA is

(3) $\pi_{i}= \begin{cases}I+v_{i} & \text { if } \sum_{j=1}^{N} c_{j} \geq P P, U P \text { exists, and } c_{i}<U P \\ I-U P+v_{i} & \text { if } \sum_{j=1}^{N} c_{j} \geq P P, U P \text { exists, and } c_{i} \geq U P \\ I & \text { otherwise }\end{cases}$

where $U P=\min \left\{p>0|n p=P P, n=|\left\{i \in\{1, \ldots, N\}: c_{i} \geq p\right\} \mid\right\}$. Note that the existence of a $U P$

implies $\sum c_{i} \geq P P$, but the converse is not true: if the contributions in aggregate exceed $P P$, but cannot satisfy $n p=P P$ for some $n=1, \ldots, N, U P$ does not exist and the mechanism does not provide; UPA is not efficient.

If an agent contributes less than $U P$, she pays nothing and the full $c_{\mathrm{i}}$ will be rebated. If an agent contributes $U P$ or more, she will pay only the price $U P$ and the excess contribution will be rebated. To provide the good, UPA requires not only that the total contribution meet or exceed 
$P P$, but also that the number of relatively high individual contributions be sufficient. More precisely, $P P$ and the group size together determine a set of at most $N$ possible prices, where $P P$ is shared by $n \leq N$ agents offering at least $P P / n$. Using the example in Section 2.2, $c=\{11,8,6\}$ will result in a $U P$ of 4 and a rebate profile of $\{7,4,2\}$; while $c=\{10,5,3\}$ with a sum of 18 which is greater than $P P$ does not support any $U P$.

\subsection{Uniform Price Cap (UPC)}

UPC is a modified version of UPA that ensures the good can be provided whenever total contributions exceed $P P$. The payoff under UPC is

(4) $\pi_{i}= \begin{cases}I-c_{i}+v_{i} & \text { if } \sum_{j=1}^{N} c_{j} \geq P P \text { and } c_{i}<U C \\ I-U C+v_{i} & \text { if } \sum_{j=1}^{N} c_{j} \geq P P \text { and } c_{i} \geq U C \\ I & \text { otherwise }\end{cases}$

where $U C=\min \left\{p>0: \sum_{j \in\left\{k c_{k}<p\right\}} c_{j}+n p=P P, n=\left|\left\{i: c_{i} \geq p\right\}\right|\right\}$. Under UPC, if there are excess contributions, a uniform price cap $(U C)$ will be calculated. If an agent contributes less than $U C$, she pays her full contribution $c_{\mathrm{i}}$ (under UPA they would pay nothing). If an agent contributes $U C$ or more, she pays only the price cap and the excess contribution is rebated, just like under UPA. $U C$ is calculated as the lowest price that could collect only the exact amount needed. Since the contributions lower than the price will not be rebated, the uniform cap $U C$ always exists as long as $P P$ is met or exceeded; UPC is efficient. Using the examples in Section $2.3, c=\{11,8,6\}$ will result in a $U C$ of 4 and a rebate profile of $\{7,4,2\} ; c=\{10,5,3\}$ will result in a $U C$ of 4.5 and a rebate profile of $\{5.5,0.5,0\}$, in contrast to non-provision under UPA.

\section{Theoretical Benchmarks: Nash Equilibrium and Marginal Penalty}

To predict how the four mechanisms will affect individual and group contributions, we use undominated perfect equilibrium (UPE) and the marginal penalty associated with contribution beyond the provision point as theoretical benchmarks. UPE makes a precise prediction about group contributions, but supports a broad continuum of equilibrium contribution profiles leading to that aggregate. We use the marginal penalty to understand patterns of disequilibrium, which may organize systematic behavior in the process of players coordinating among multiple equally refined UPE. To simplify the discussion, we will focus on the case where it is efficient to provide the public good, but no one would like to provide the good alone and no one is pivotal in provision: in PPM, PR, and UPC, this requires that $\sum_{j} v_{j}-v_{i}>P P>v_{i}$ for all $i=1, \ldots, N$; in 
UPA, it means that the value profile of the $N$ agents supports at least one uniform price $U P$ and that dropping the value of one agent does not preclude provision, formally that $\left\{P P / k: k \in\{1, \ldots, N\},\left|\left\{j \in\{1, \ldots, N\} \backslash\{i\}: v_{j} \geq P P / k\right\}\right| \geq k\right\} \neq \varnothing$ for all $i=1, \ldots, N$.

\subsection{Undominated Perfect Equilibrium}

We first define the notion of undominated perfect equilibrium (UPE). Our provision point mechanism and its variations have a continuous strategy space, but following Bagnoli and Lipman (1989), we instead apply trembling hand perfection to a sequence of approximating games, where the strategy space is discretized into a sequence of intervals $\left\{\mu_{n}\right\}$, with the grid size $\mu_{n} \downarrow 0$ as $n \rightarrow \infty$. Specifically, given an interval $\mu_{n}$, for each agent $i$, a smallest element $a_{i}(n)$ is chosen within $\mu_{n}$ from the lower bound of $S_{i}$, and the finite strategy set $S_{i}(n)$ consists of all the points of the form $a_{i}(n)+k \mu_{n}$ which are less than the largest element of $S_{i}$ for any nonnegative integer $k$. Then a strategy profile is a perfect equilibrium of a mechanism on $S$ if it is the limit of a sequence of perfect equilibrium points of a sequence of approximating finite games on $S(n)$, where $S=S_{1} \times \cdots \times S_{N}$. Note, a perfect equilibrium on a finite strategy set is a strategy profile $c \in S(n)$ such that $c_{i} \in S_{i}(n)$ is a best reply to each point in a sequence of completely mixed strategies for the other players converging to $c_{-i} \in S_{-i}(n)$, where $-i$ indicates agents other than $i$, and a completely mixed strategy assigns a strictly positive probability to each of the supporting pure strategies.

Further, we say a strategy for $i, c_{i} \in S_{i}$, is dominated if there exists $c_{i}^{\prime} \in S_{i}$ such that for all $c_{-i} \in S_{-i}, \pi_{i}\left(c_{i}^{\prime}, c_{-i}\right) \geq \pi_{i}\left(c_{i}, c_{-i}\right)$, with a strict inequality for some $c_{-i} \in S_{-i}$. Let $R\left(S_{i}\right)$ denote the set of strategies for agent $i$ which are not dominated. Then, we define an undominated perfect equilibrium of a mechanism as a perfect equilibrium on $R(S)=R\left(S_{1}\right) \times \cdots \times R\left(S_{N}\right)$.

The UPE concept eliminates dominated strategies before refining by trembling hand perfection. Bagnoli and Lipman (1989) introduce the refinement of undominated perfection to eliminate all non-provision equilibria and show that the set of strategy profiles such that the provision point is exactly met and no one contributes more than their value, $v_{\mathrm{i}}$, are the UPE of PPM with complete information; these UPE are Pareto efficient. They also argue that, when rebate rules are incorporated into PPM, as long as the rebate scheme has the property that 
increasing one's contribution by $\$ 1$ never increases one's rebate by more than $\$ 1$, the resulting game has the same set of UPE. PR and UPC both satisfy this property and have the same condition for provision as PPM. We show in the appendix that PR and UPC have the same UPE as PPM, but with an additional regularity condition. The regularity condition excludes perverse cases that lead to individual payoffs of zero in the event of provision, in order to eliminate all non-provision equilibria. ${ }^{4}$ The UPE outcomes of PR and UPC are summarized in Proposition 1. Proposition 1. Subject to regularity condition A.1, with complete information, if $\sum_{j} v_{j}-v_{i}$ $>P P>v_{i}$ for all $i=1, \ldots, N$, for PPM, PR, and UPC, the set of strategy profiles such that $\sum_{i} c_{i}=P P$ and $c_{i} \leq v_{i}$ for all $i$, is the set of undominated perfect equilibria.

Proof. See appendix.

UPA has a different set of UPE from the other three mechanisms. Bagnoli and Lipman (1989) also require that the only condition of provision be that $P P$ is met or exceeded, while UPA imposes constraints on the configurations of contributions that aggregate to meet the provision condition. We characterize the UPE set of UPA in Proposition 2.

Proposition 2. Subject to regularity condition A.2, with complete information, if for all $i=1, \ldots$, $N, \sum_{j} v_{j}-v_{i}>P P>v_{i}$, and $\left\{P P / k: k \in\{1, \ldots, N\},\left|\left\{j \in\{1, \ldots, N\} \backslash\{i\}: v_{j} \geq P P / k\right\}\right| \geq k\right\} \neq \varnothing$, for UPA, the set of strategy profiles such that one and only one uniform price can be set and $c_{i}<\min \left\{P P / k>v_{i}: k \in\{1, \ldots, N\}\right\}$ for all $i$, is the set of undominated perfect equilibria.

Proof. See appendix.

Note that when it is socially efficient and feasible to provide the public good, the UPE outcome of UPA is the same as that of the other three mechanisms, in the sense that the good will be provided and the actual total payment is equal to the provision cost. However, since the UPE of UPA is based on the possible uniform prices instead of group contributions, UPA's set of equilibria differs from the other mechanisms in two important ways. First, group contributions above $P P$ are supported as equilibria. The only condition under which a uniform price $U P$ exists when the provision point is exactly met is when $n=P P / U P$ is an integer number of agents each

\footnotetext{
${ }^{4}$ In Bagnoli and Lipman (1989), a strictly positive individual payoff in the case of provision is necessary in order to eliminate all non-provision equilibria by the refinement of trembling hand perfection, which is guaranteed by the notion of undominated for PPM. For PR, UPC, and UPA, however, zero individual payoffs are still possible under undominated perfection for some knife-edge cases that are unlikely to arise in application, which we eliminate through additional regularity conditions. See appendices for Propositions 1 and 2 for details
} 
contributing $U P$ and the other $N-(P P / U P)$ agents choose $c_{\mathrm{i}}=0$. There are at most $N$ cases of UPE satisfying this condition; other UPEs of UPA involve group contributions strictly higher than PP. Second, the UPE of UPA does not exclude $c_{\mathrm{i}} \mathrm{s}$ that are greater than $v_{\mathrm{i}}$ (which are dominated in other mechanisms), as long as the corresponding payments will not exceed $v_{\mathrm{i}}$ under any tremble. A contribution $c_{\mathrm{i}}$ above $v_{\mathrm{i}}$ is undominated as long as $c_{\mathrm{i}}$ is less than the lowest possible price higher than $v_{\mathrm{i}}$. These two properties imply that the UPE of UPA includes aggregate and individual contributions that are not supported in the UPE of the other three mechanisms.

While the UPE refinement makes distinct predictions for UPA and the other mechanisms, it is inadequate in two ways. First, equilibrium is not strongly predictive in existing PPM and PR experiments: Bagnoli and McKee (1991) report that the provision point is exactly met in only 54\% of their PPM periods with five homogeneous subjects; Marks and Croson (1998) report 34\% in PPM and 7\% in PR. Second, there is still a wide continuum of individual UPE strategies in each mechanism, and three mechanisms have the same equilibrium strategy set, and so UPE alone cannot explain differences in contributions among mechanisms. Therefore, we use the marginal penalty as a second theoretical benchmark.

\subsection{Marginal Penalty of Overcontribution}

The marginal penalty of overcontribution captures the private payoff loss associated with an additional unit of contribution, conditioned on provision. Marks and Croson (1998) argue that aggregate contributions will be higher when the marginal penalty is lower. Given the continuum of provision UPE of each mechanism, coordination on any particular equilibrium may be difficult in practice. Most of the equilibria are asymmetric, and involve some agents contributing "more than their share" to compensate for the low contributions of the others. The coordination problem has two important implications. First, excess contributions are likely when provision occurs. Second, mechanisms that punish excess contributions may thwart efforts to coordinate on such an asymmetric equilibrium, which may lower the observed rate of provision.

Figure 1 shows individual payoffs and the structures of marginal penalty for agent $i$ under different mechanisms, conditioned on provision. For PPM (Panel A), since excess contributions will not be rebated, every dollar contributed to the public good beyond $P P$ will be burned, and thus the marginal penalty of overcontribution is -1 . For PR (Panel B), the marginal penalty is $\partial \pi_{i} / \partial c_{i}=-P P \cdot C_{-i} / C^{2}$ (Marks and Croson, 1998), where $C=\sum_{j} c_{j}$ and $C_{-i}=\sum_{j \neq i} c_{j}$. This 
is bounded between -1 and 0 , and almost always greater than -1 . Marks and Croson argue the lower marginal penalty will lead to higher contributions in PR than PPM.

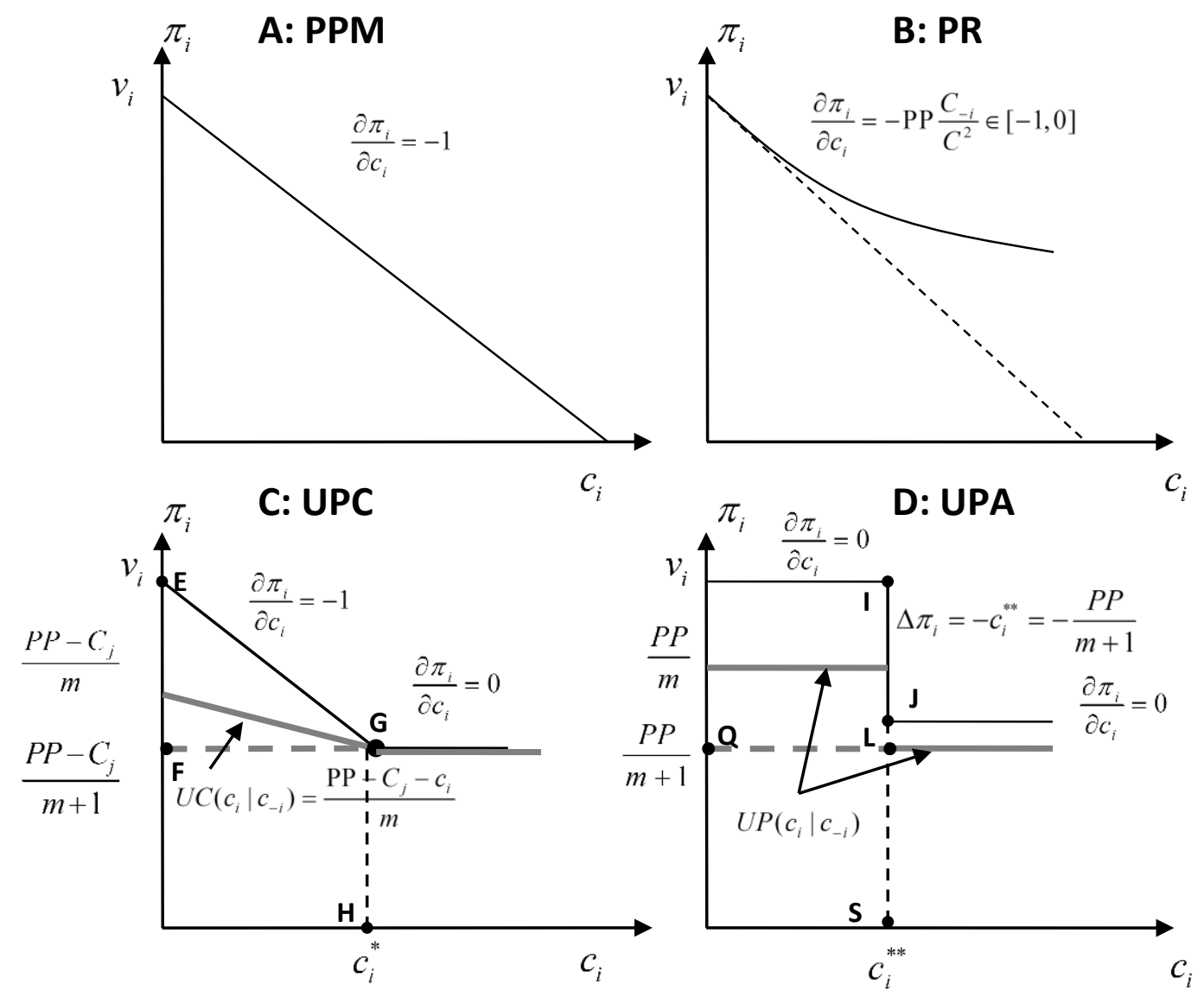

Figure 1 Individual Payoff and the Structures of Marginal Penalty, Conditioned on Provision Note: This figure shows how agent $i$ 's payoff, $\pi_{\mathrm{i}}$, changes when $i$ 's contribution, $c_{\mathrm{i}}$, increases from 0 , conditioned on the good

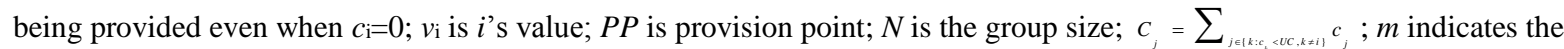
number of agents contributing at or above the uniform price in UPC or UPA excluding agent $i$; $U C\left(c_{\mathrm{i}} \mid c_{-\mathrm{i}}\right)$ and $U P\left(c_{\mathrm{i}} \mid c_{-\mathrm{i}}\right)$ are respectively uniform cap and uniform price as a function of $c_{\mathrm{i}}$ given the others' contributions. $c_{\mathrm{i}}{ }^{*}$ and $c_{\mathrm{i}}{ }^{* *}$ are the cutting points respectively for $\mathrm{UPC}$ and $\mathrm{UPA} ; \mathrm{EF}=\mathrm{FG}=\mathrm{GH}$ if assuming no price below $\left(P P-C_{j}\right) /(m+1)$ is possible in $\mathrm{UPC}$; $\mathrm{IJ}=\mathrm{QL}=\mathrm{LS}$; the initial endowment is set to be zero for convenience.

Our uniform price mechanisms aim to increase contributions by creating regions of zero marginal penalty. In UPC, if $c_{\mathrm{i}}$ is at or above $U C\left(c_{\mathrm{i}} \mid c_{-\mathrm{i}}\right)$, the level of the cap that provides the good as a function of $c_{\mathrm{i}}$ given $c_{\text {-i }}$, then any incremental contribution will not change the uniform price and will be fully rebated, creating a marginal penalty of 0 . If $c_{\mathrm{i}}<U C\left(c_{\mathrm{i}} \mid c_{-\mathrm{i}}\right)$, the marginal penalty is illustrated in Figure 1 (Panel C). Here, there exists a cutpoint, $c_{\mathrm{i}}{ }^{*}$, at which the 
marginal penalty changes from - 1 to 0 . In Figure 1, the intercept of the grey solid line with the $y$ axis represents the realized uniform price when $c_{\mathrm{i}}=0$. When approaching $c_{\mathrm{i}}{ }^{*}$ from below, $U C\left(c_{\mathrm{i}} \mid c_{-\mathrm{i}}\right)$ decreases and the increased contribution is fully collected, leading to a marginal penalty of -1 ; when approaching $c_{\mathrm{i}}{ }^{*}$ from above, $U C\left(c_{\mathrm{i}} \mid c_{-\mathrm{i}}\right)$ stays constant at $c_{\mathrm{i}}{ }^{*}$ and the marginal penalty is 0 ; at $c_{\mathrm{i}}=c_{\mathrm{i}}{ }^{*}$, the marginal penalty is not defined. Based on beliefs about $c_{\text {-i }}$, agents calculate an expected marginal penalty between -1 and 0 , implying higher group contributions in UPC than in PPM, while the ranking between UPC and PR would depend on beliefs.

The marginal penalty structure of UPA is similar to that of UPC, but with a critical difference. If $c_{\mathrm{i}}<U P\left(c_{\mathrm{i}} \mid c_{-\mathrm{i}}\right)$ (Figure 1, Panel D) where $U P\left(c_{\mathrm{i}} \mid c_{-\mathrm{i}}\right)$ is the uniform price that provides the good through payments of $P P / m$ by $m$ other agents, there exists a cutpoint, $c_{\mathrm{i}}{ }^{* *}$, at which $i$ 's contribution is sufficient to be included in payments of the next lowest uniform price, and the final payment by $i$ jumps from 0 to the new price, $U P\left(c_{\mathrm{i}}^{* *} \mid c_{-\mathrm{i}}\right)=P P /(m+1)$. At all other points, the marginal penalty is 0 , even when the contribution is lower than $c_{\mathrm{i}}{ }^{* *}$. Thus, the marginal penalty of UPA is zero almost always, except at the cutpoint with a lump sum penalty. Given the broad range of values with no marginal penalty, we conjecture higher contributions in UPA than the other mechanisms.

Note that the marginal penalty structures of UPC and UPA not only suggest differences in aggregate contributions among mechanisms, but also if agents with higher $v_{\mathrm{i}}$ s tend to make higher contributions, they are also suggestive of how incentives may differ across the range of values: agents with higher $v_{\mathrm{i}}$ s may make higher contributions in UPC and UPA than in PPM and $\mathrm{PR}$, because the marginal penalty for contributions typical of their value range is lower. Similarly, we would also expect a higher contribution level from low value agents in UPA than in the other mechanisms, while the contribution level from low value agents in UPC could be higher or lower than that from PPM or PR.

These theory based-benchmarks provide the basis for hypotheses we test in the experiment described below. In particular, we make the following predictions:

1) Contributions: With the largest set of contributions supported as UPE, and zero marginal penalty across the largest range of the contribution space, UPA generates the highest contributions. With a large range with zero marginal penalty, UPC generates more contributions than PR, and PPM has the lowest contribution level. At the individual level, 
UPA induces the highest contributions at all value ranges while UPC induces higher contributions than PPM and PR only at high values.

2) Provision rate: With higher contributions and the same provision rule, UPC will have the highest contribution rate, followed by PR, followed by PPM. Due to the restrictions on uniform prices, UPA will have the lowest provision rate, despite higher contributions.

\section{Experimental Design and Procedures}

To test the predictions of UPE, and the effects of marginal penalty, on the four mechanisms, we designed a controlled laboratory experiment in which subjects make contributions toward an induced value public good under complete information. Table 1 shows the mechanism treatments presented in each session: the first set of 3 treatments uses homogeneous induced values, and the second heterogeneous values. Within each set, the first treatment is always PPM (25 periods), to familiarize subjects with the baseline game; the following two treatments (25 periods each) apply the other mechanisms in a Latin Square to control for order effects.

Table 1 Treatment Arrangement of Experimental Sessions

\begin{tabular}{c|ccc|ccc}
\hline \multirow{2}{*}{$\begin{array}{c}\text { Treatment } \\
\text { Order }\end{array}$} & \multicolumn{2}{|c|}{$\begin{array}{c}\text { Homogeneous Induced Values } \\
\text { (2 groups of size 5) }\end{array}$} & \multicolumn{3}{|c}{$\begin{array}{r}\text { Heterogeneous Induced Values } \\
\text { (1 group of size 10) }\end{array}$} \\
\cline { 2 - 7 } & $\begin{array}{c}\text { 1st } \\
(25 \mathrm{pds})\end{array}$ & $\begin{array}{c}\text { 2nd } \\
(25 \mathrm{pds})\end{array}$ & $\begin{array}{c}\text { 3rd } \\
(25 \mathrm{pds})\end{array}$ & $\begin{array}{c}4 \text { th } \\
(25 \mathrm{pds})\end{array}$ & $\begin{array}{c}5 \text { th } \\
(25 \mathrm{pds})\end{array}$ & $\begin{array}{c}\text { 6th } \\
(25 \mathrm{pds})\end{array}$ \\
\hline Session 1 & PPM & PR & UPC & PPM & PR & UPC \\
Session 2 & PPM & PR & UPA & PPM & PR & UPA \\
Session 3 & PPM & UPC & PR & PPM & UPC & PR \\
Session 4 & PPM & UPC & UPA & PPM & UPC & UPA \\
Session 5 & PPM & UPA & PR & PPM & UPA & PR \\
Session 6 & PPM & UPA & UPC & PPM & UPA & UPC \\
\hline
\end{tabular}

The homogeneous value treatments, where each subject is assigned $v_{\mathrm{i}}=10$, have 2 groups of 5 subjects randomly regrouped among 10 session participants in each period, providing baseline comparisons among mechanisms. This setup is similar to that in Marks and Croson (1998), except we regroup each period to maintain the equilibrium selection problem that gives the best chance to observe the effects of marginal penalty differences among the mechanisms. The heterogeneous value treatments are designed to induce a wider range of contributions within each mechanism, to provide a yet stronger test of the marginal penalty effect. To have a wide 
range of possible uniform prices, we pool the 10 session participants in one group, ${ }^{5}$ and assign one of the 10 members exclusively to one of the 10 induced values $\{4,4,5,5,6,6,8,8,10,12\}$, with the value-assignment reshuffled among group members in each period. The provision point is 30 in all treatments. These experimental parameters are chosen in a way that providing the good is always socially optimal, and no induced value is lower than the lowest UPA prices. All the information above is common knowledge.

The experimental software was developed in Z-Tree (Fischbacher, 2007). At the start of each treatment, the experimenter read the instructions aloud as subjects read along. Subjects were then given an initial budget of 15 experimental dollars. Subjects then simultaneously choose a contribution, $c_{\mathrm{i}} \in[0,15]$ (with a precision of 0.1 ) towards the project. At the end of each period, subjects were informed whether the project is provided, and their earnings, payment and rebates. At the end of a session, earnings were totaled across all periods. Subjects were recruited through university-wide daily digest email server (mainly for undergraduates), and from an email list of students interested in participating in experiments. A total of 60 subjects participated in the six sessions, producing 9000 individual-level observations. Each session lasted roughly 1.5 hours, with an average payment of $\$ 25$.

\section{Results}

We measure the performance of the mechanisms by two indicators: aggregate group contribution and the provision rate. The provision rate reflects the efficiency of the mechanism, as provision is always efficient given our parameter values. Group contribution allows us to identify the role of marginal penalties, which are meaningful only when excess contributions are observed. In addition, group contribution is a measure of the extent of value revelation, which is of interest in cases where small-scale real money, real good provision point mechanisms are used to provide estimates of public value for non-market goods that are then applied over a broader population (e.g., Champ et al, 2002; Haskell et al., 2010; Bush et al., 2013; Swallow, 2013)

Figure 2 gives an overview of group contributions in each period, and five-period average provision rates, by mechanism in the homogeneous and heterogeneous induced value

\footnotetext{
${ }^{5}$ Our objective here is not to test for group size or heterogeneous value effects per se, but to provide a stronger test of differences among mechanisms in what we expect will be a more discriminating environment.
} 
environments. Grey lines represent session-specific group contributions, dark lines represent averages over sessions, and dark dots represent five-period provision rates. ${ }^{6}$

\subsection{Group Contributions}

Consistent with our hypotheses, the uniform price mechanisms induce higher contributionsmuch higher in the case of UPA. In the homogeneous environment, UPC is also consistently above the provision point, PR oscillates around it, while PPM is consistently below it. In the heterogeneous environment, UPC is slightly higher than PR, and both of them are higher than PPM, which is in the neighborhood of the provision point. Importantly, across mechanisms and treatments, group contributions are generally not equal to the provision point and do not stabilize period-to-period, especially in the heterogeneous environment, reflecting the coordination problem.

\footnotetext{
${ }^{6}$ Each session-specific group contribution in a period in the homogeneous environment is the average contribution of two groups of size 5, since group members are reshuffled among 10 individuals in each period.
} 


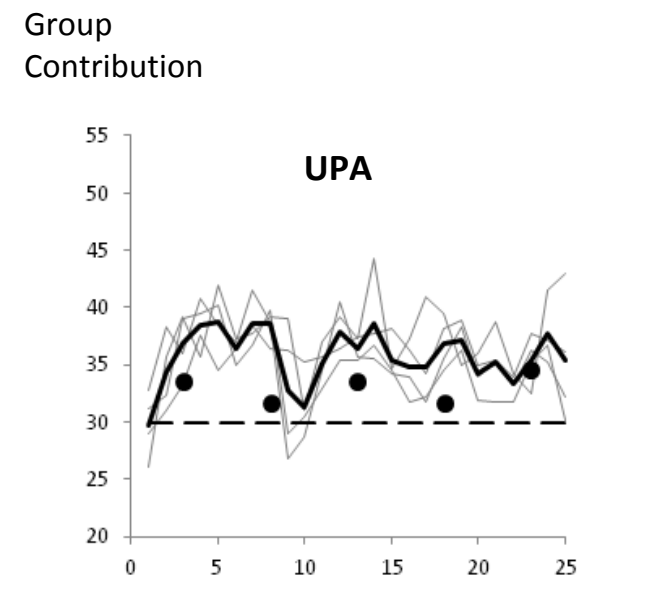

Group

Contribution

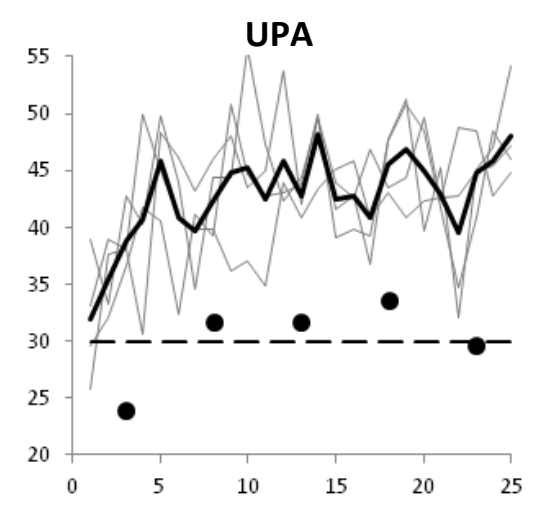

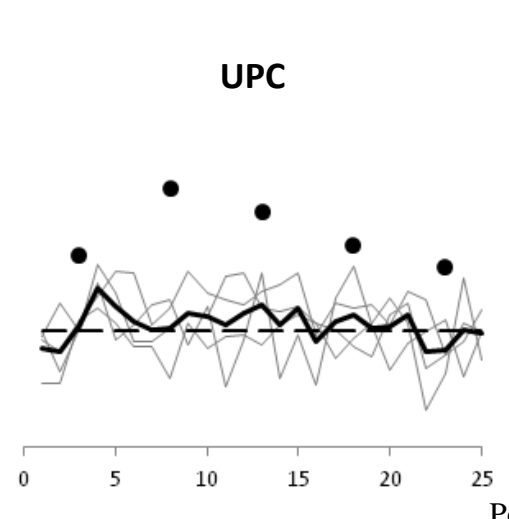

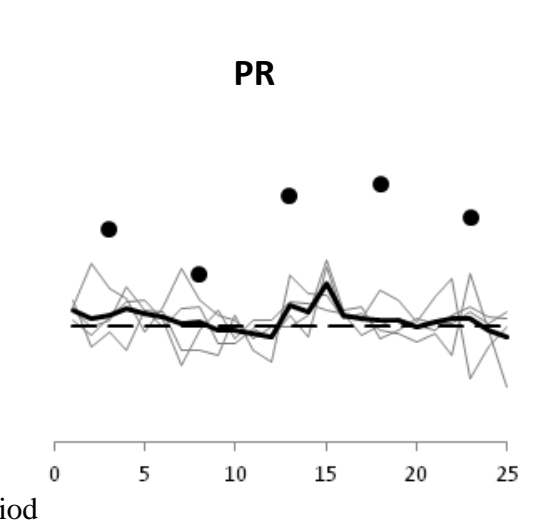

Heterogeneous Induced Values

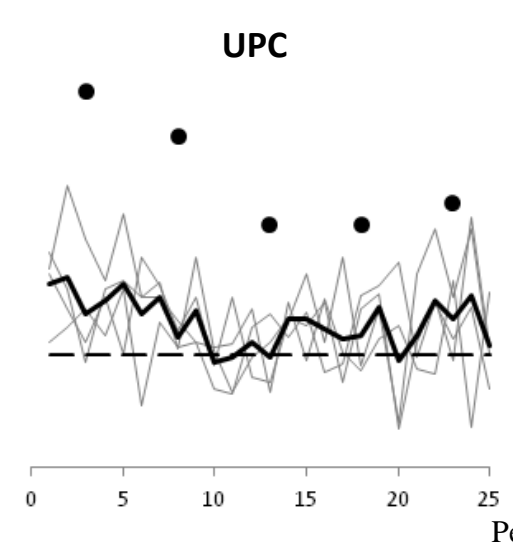

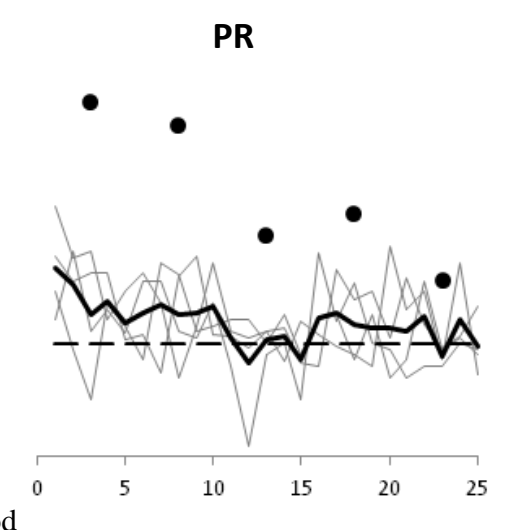

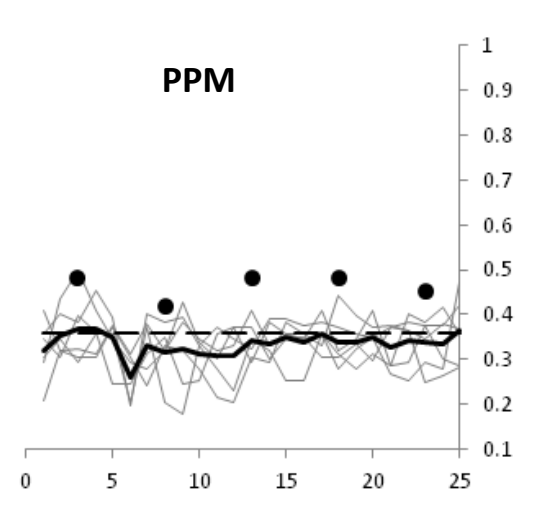

Five-period Provision Rate

Session Group Contribution ---- Provision Point

Mean Group Contribution - Provision Rate

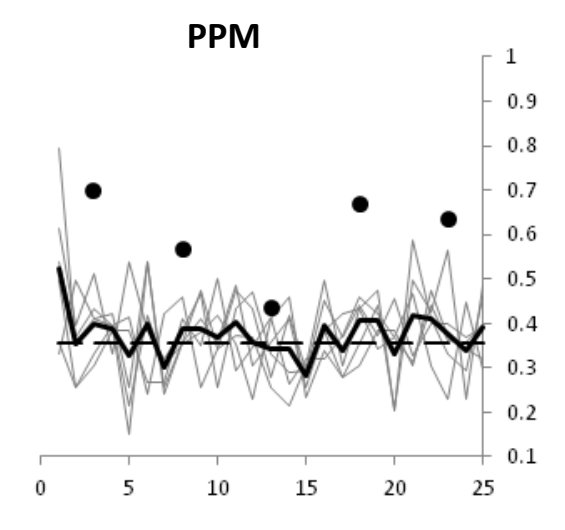

Figure 2 Group Contributions in Each Period and 5-Period Provision Rate by Mechanism 
Table 2 presents results from two-factor random effects regressions of group contributions in the last 20 periods of each treatment on mechanism dummies (group- and period-specific, cf. Marks and Croson, 1998). ${ }^{7}$ Models 1 and 3 each provide a baseline that includes only mechanism dummies, using PPM as the base, for groups with homogeneous and heterogeneous values. Models 2 and 4 control for the previous two periods' provision rate, reflecting a Cournot-type response dynamic to minimize private costs based on previous provision outcomes (cf. Issac et al. (1989)'s notion of cheap riding). ${ }^{8}$ Likelihood ratio tests advise using Models 2 and 4 for mechanism comparisons.

Table 2 Two-factor Random Effects Models of Group Contribution

\begin{tabular}{lllll}
\hline \multirow{2}{*}{ Group Contribution } & \multicolumn{2}{l}{ Homogeneous Values } & \multicolumn{2}{l}{ Heterogeneous Values } \\
& $(1)$ & $(2)$ & $(3)$ & $(4)$ \\
\hline PR & $0.993^{* * *}$ & $0.925^{* * *}$ & 0.815 & $1.035^{*}$ \\
& $(0.346)$ & $(0.344)$ & $(0.542)$ & $(0.538)$ \\
UPC & $1.891^{* * *}$ & $1.955^{* * *}$ & $1.730^{* * *}$ & $2.036^{* * *}$ \\
& $(0.346)$ & $(0.344)$ & $(0.542)$ & $(0.542)$ \\
UPA & $7.053^{* * *}$ & $8.079^{* * *}$ & $13.37^{* * *}$ & $13.03^{* * *}$ \\
& $(0.346)$ & $(0.545)$ & $(0.542)$ & $(0.544)$ \\
Provision Rate ${ }^{\dagger}$ & & & & $-1.955^{* * *}$ \\
& & & & $(0.594)$ \\
Provision Rate $\times$ UPA & & $-2.387^{* * *}$ & & \\
Constant (PPM) & & $(0.990)$ & & \\
& $28.97^{* * *}$ & $28.97^{* * *}$ & $30.47^{* * *}$ & $31.59^{* * * *}$ \\
\hline Log-likelihood & $(0.442)$ & $(0.469)$ & $(0.343)$ & $(0.480)$ \\
Chi-square & -823.3 & -820.5 & -986.9 & -981.6 \\
$\mathrm{R}^{2}$ overall & 435.2 & 448.4 & 716.8 & 749.2 \\
Number of observations & 0.503 & 0.499 & 0.666 & 0.675 \\
Number of periods (treatment-specific) & 360 & 360 & 360 & 360 \\
\hline St & 20 & 20 & 20 & 20 \\
\hline
\end{tabular}

Standard errors in parentheses; $* * * \mathrm{p}<0.01, * * \mathrm{p}<0.05, * \mathrm{p}<0.1$;

$\dagger$ : Provision rate over previous 2 periods, which yields the largest log-likelihood among 1 to 5 -period lags.

\footnotetext{
${ }^{7}$ The two-factor random effects models are based on the following regression: $y_{i t}=X_{i t} \beta+u_{i}+v_{t}+\varepsilon_{i t}$, where $y_{i t}$ represents the group contribution for group $i$ in period $t$, with the two random effects denoted by $u_{i}$ and $v_{t}$, respectively, and $X_{i t}$ is a set of regressors including mechanism dummies PR, UPC, and UPA with PPM as the baseline, the previous two periods' provision rate denoted by Provision Rate in Table 2, and an interaction term of the provision rate with the UPA dummy. As explained in the previous footnote, the average contribution is used in the homogenous environment to be consistent with the session-group specific effect.

${ }^{8}$ Since group members are reshuffled in the homogeneous environment and induced values are reshuffled in the heterogeneous environment, the provision rate over the last several previous periods reflects how far away the average group contribution has been from the provision point. In addition, we find the provision rate effect is significant only under UPA in Model 2, while under all mechanisms in Model 4. This seems to suggest that provision rate matters only when group contributions are not only statistically but also economically higher than the provision point, which makes sense since it may be too risky to "cheap ride" if group contributions are not way above the provision point.
} 
With $P P=30$, the regression constant in each model represents the model prediction of the average group contribution under PPM, and the mechanism dummies represent the mechanismspecific differences from PPM. Models 2 and 4 support our predictions on group contributions: the ordering of the mechanisms is broadly consistent with higher contributions occurring where the expected marginal penalty is lower. UPA - with an almost-everywhere zero marginal penalty and expanded equilibrium outcome set (supporting contributions above $P P$ ) - is significantly higher than the others all with $p<0.001$ in both environments. Similarly, the lower expected marginal penalty from UPC leads to significantly higher group contributions than PPM and PR in both environments. ${ }^{9}$ PR also generates contributions significantly higher than PPM, due to a smaller marginal penalty. ${ }^{10}$

These results contrast with Marks and Croson (1998), who find group contributions under PPM and PR are not statistically distinguishable. This may be attributable to the more challenging coordination process in our experiment providing a stronger test of the mechanisms: in their design, fixed groups of five homogeneous subjects were able to stabilize their total contributions at the provision point over 25 periods, while our groups were reshuffled every period, maintaining the strategic uncertainty and allowing the coordination problem to persist. Marks and Croson's group contributions are not significantly different from $P P$ under both PPM and PR, based on a regression similar to our Model 1. However, in our data, aggregate contributions are generally not equal to the UPE predicted level of PP: in Model 1, the average group contribution is significantly different from 30 in PPM $(28.97 ; p=0.020)$, and also borderline different in UPC (30.86; $p=0.070)$; similarly, in PR $(31.28, p=0.002)$ and UPC (32.20, $\mathrm{p}<0.001)$ in Model 3. Although the group contributions in UPA-significantly higher than PP (homogeneous values: 36.02, $p<0.001$; heterogeneous values: 43.84, $p<0.001$ ) —are consistent with the UPE prediction for that mechanism, they still indicate that subjects cannot efficiently coordinate around one of a few possible prices, or even a focal symmetric price (6) in the homogeneous environment. Therefore, our environment is more powerful for distinguishing among the mechanisms, and provides a better testbed for determining how the marginal penalty story enhances the UPE prediction, and whether the uniform price mechanisms represent a practical improvement over PPM and PR for some applications.

\footnotetext{
${ }^{9}$ Model 2: PPM vs. UPC ( $\left.p<0.001\right)$, PR vs. UPC ( $\left.p=0.010\right)$; Model 4: PPM vs. UPC ( $\left.p<0.001\right)$, PR vs. UPC $(p=0.087)$.

${ }^{10}$ Model 2: PPM vs. PR ( $\left.p=0.007\right)$; Model 4: PPM vs. PR $(p=0.054)$.
} 


\subsection{Provision Rate}

With the same provision condition among UPC, PR and PPM, the higher contributions associated with a lower expected marginal penalty generally result in a higher provision rate (Figure 2 dots), consistent with our predictions. UPC — designed with marginal penalty in mind - performs weakly better than PR in the sense that UPC is significantly better than PPM in the heterogeneous environment while PR is not. Specifically, UPC has provision rates significantly higher than those for PPM in both environments (homogeneous: $58.8 \%$ vs. $45.8 \%$, $z$-test $p=0.011$; heterogeneous: $71.3 \%$ vs. $57.5 \%, p=0.049)$. PR is significantly higher than PPM only with homogeneous values $(60.0 \%, p=0.006$; heterogeneous: $65.0 \%, p=0.288)$. With comparable expected marginal penalties, UPC and PR have similar provision rates in both environments ( $p=0.820$ and $p=0.396$ ). Because the profile of offers, in addition to the total amount, affects the provision decision in UPA, it has lower provision rates than the other mechanisms in both homogeneous (43.1\%) and heterogeneous (40.0\%) environments, all with $p<0.01$ except for PPM $(45.8 \%, p=0.594)$ in the homogeneous environment.

To understand how a lower expected marginal penalty facilitates coordination, and ultimately induces higher group contributions and provision rates, we look into the differences among mechanisms at the individual contribution level. Figure 3 shows the cumulative distributions of individual contributions by mechanism in the homogeneous and heterogeneous environments; these graphs can be interpreted as the selected strategy profiles. ${ }^{11}$

\subsection{Distributions of individual contributions with homogeneous induced values}

With homogeneous values, the observed distributions of contributions show how a wide continuum of efficient equilibria incentivizes people to deviate from the symmetric equilibrium $\left(c^{*}=6\right)$ in hopes of favorably asymmetric cost sharing: observed individual contributions in all mechanisms have a wide range, inconsistent with playing the focal symmetric equilibrium, although the symmetric contribution of six is indeed the mode in all mechanisms (Figure 3, upper panel). This reveals the fundamental coordination problem of how much above six to contribute for subjects who are willing to compensate for those deviating below. With successful coordination admitting the possibility of excess group contributions attributable to the above- 6 contributors, no rebate (-1 marginal penalty) may hurt the coordinating incentive, even to the point where average group contribution is below $P P$ for homogenous PPM.

\footnotetext{
${ }^{11}$ In the heterogeneous environment, the distribution is over individual contributions from pooled induced values.
} 
Therefore, the role of a lower expected marginal penalty in facilitating coordination is to reduce the commitment associated with offering to coordinate on disadvantageous asymmetric equilibria, making the mechanism more robust to cheap riding. For conditional cooperators, an effective marginal penalty schedule will reduce the need to determine ex ante when coordination has failed in choosing a contribution, as the mechanism can choose the desired conditional contribution after observing all contributions. In fact, all rebate mechanisms attracted more individual contributions above 6 than PPM, led by the low-marginal-penalty uniform price mechanisms (Figure 3): 50\% (z-test $p<0.001)$ for UPA, followed by UPC $(43 \%, p<0.001)$ and PR $(37 \%, p=0.041)$, all compared to PPM $(32 \%) .{ }^{12}$

\footnotetext{
${ }^{12}$ By Kolmogorov-Smirnov test, the cumulative distributions are indeed significantly different across mechanisms (PPM vs. PR with $p=0.011$; all the other pairs with $p<0.001$ ). Further, the significances are mainly due to the large differences in the distributions of contributions above the symmetric level, which drives the mechanism ranking, except for the comparison between UPC and UPA, where the significance is due to the large difference in belowsymmetric-level individual contribution.
} 

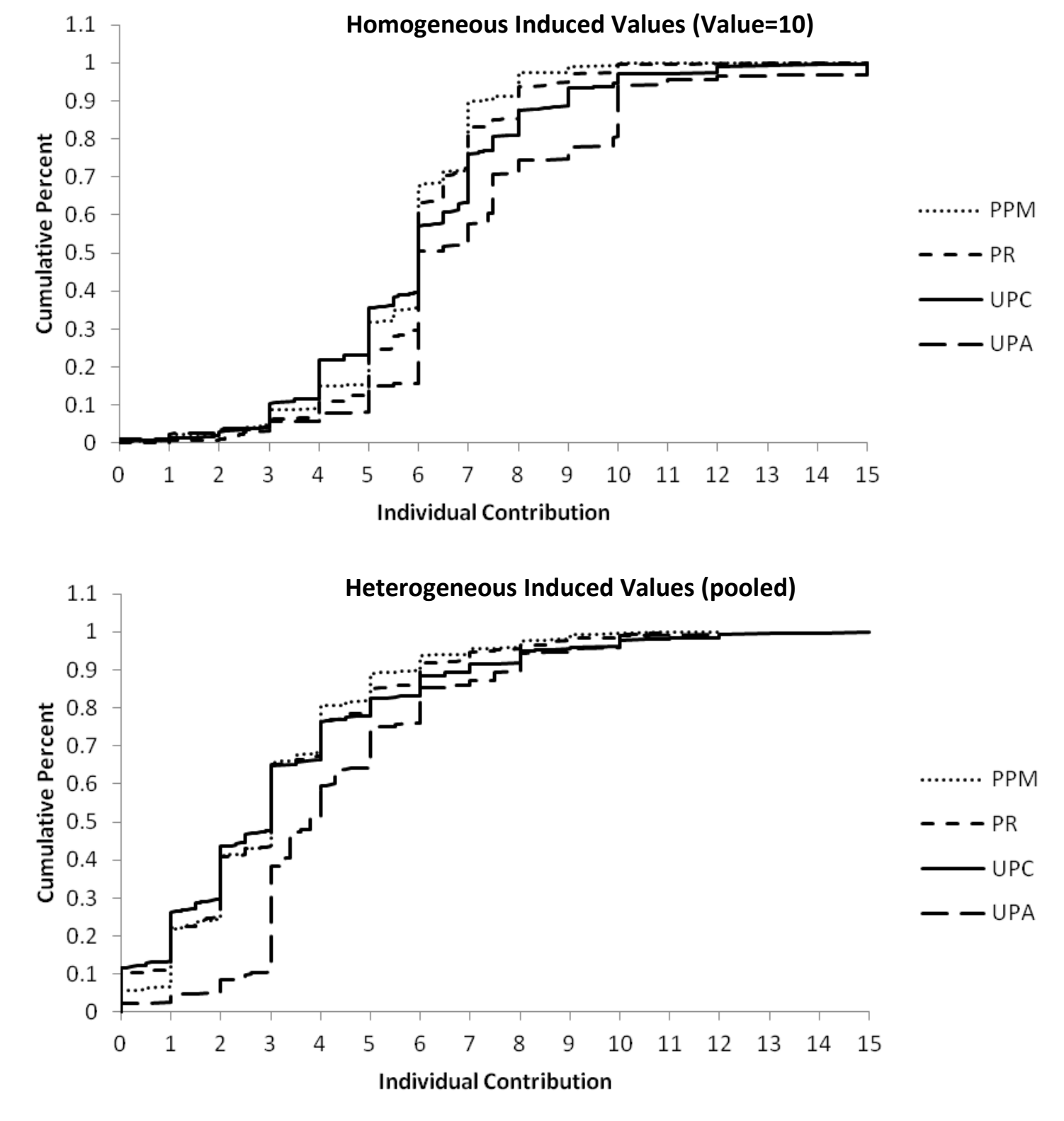

Figure 3 Cumulative Distribution of Individual Contribution by Mechanism

\subsection{Distributions of individual contributions with heterogeneous induced values}

With heterogeneous values, all rebate mechanisms generate a greater number of contributions above the symmetric UPE of 3 than PPM. They are ordered by marginal penalty, led by UPA, although the differences are smaller than in the homogeneous environment, except for UPA in the low-contribution range (Figure 3, lower panel). ${ }^{13}$ Since the actual marginal penalty observed

\footnotetext{
${ }^{13}$ By Kolmogorov-Smirnov test, UPA is significantly $(p<0.001)$ different from the others, and UPC is significantly different from PPM $(p=0.022)$ but is similar to PR $(p=0.305)$, with the latter two similar as well $(p=0.247)$, all of which are consistent with the mechanism ranking.
} 
depends on the range of the contribution, and contribution is typically a function of value in heterogeneous environments, we compare mechanisms based on the marginal penalty structures which prevail in ranges of contributions observed to be associated with different induced values. Hence, we compare individual contributions by induced value in Figure 4, where average observed uniform prices from UPA and UPC are also included to show how being close to the kink in the marginal penalty function differentiates contributions across mechanisms.

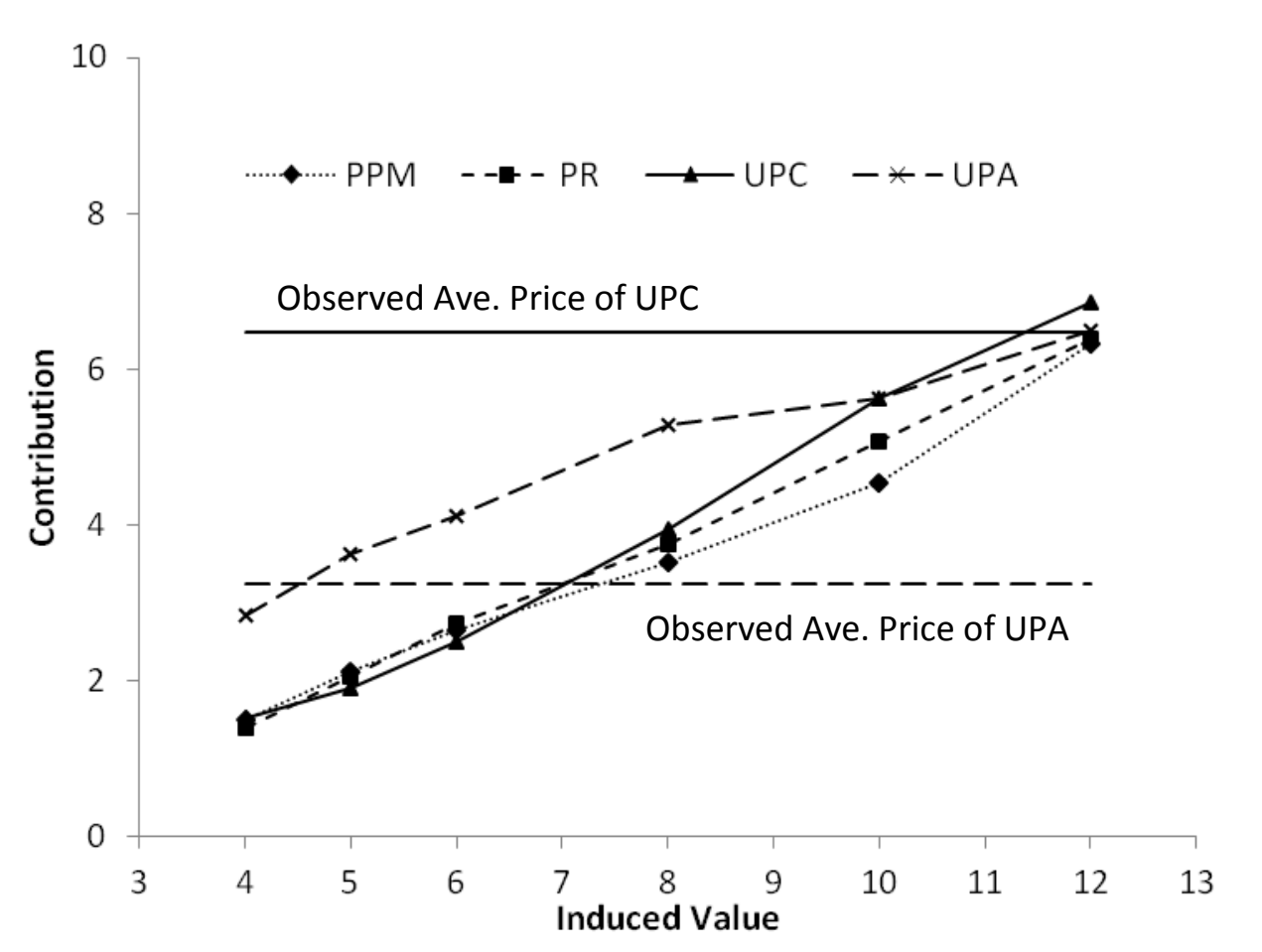

Figure 4 Mean Contributions by Induced Value

Figure 4 shows a pattern of individual contributions consistent with our predictions. Average contributions increase with induced value in all mechanisms, but they are yet higher in the uniform price mechanisms: UPA stands out as generating much higher contributions, especially at low values, and UPC generates higher contributions at high values, both of which are consistent with the distribution of contributions in Figure 3.

The patterns of UPA and UPC in Figure 4 are supported by random effects tobit regressions of individual contributions on mechanism treatment (Table 3 in the appendix); further, they reflect subjects' responding to differential marginal penalties within a mechanism across the value range. UPA's estimated contribution function has a significantly higher intercept and a significantly but slightly flatter slope than those for the other mechanisms, indicating that UPA generates higher contributions throughout the value range, more significantly at low values close 
to the observed average UPA price where a lump sum penalty occurs. With an intercept slightly lower (-0.746, $p=0.023)$ than PPM, UPC's contribution function has a significantly steeper slope (0.697) than PPM $(0.569, p<0.001)$ and PR $(0.624, p=0.003)$, which implies UPC elicits higher contributions close to its observed uniform price than do PPM and PR, and above which the expected marginal penalty in UPC is lower. ${ }^{14}$ PR generates meaningfully higher contributions than PPM only at the highest values, so differences in aggregate contributions are insignificant between PR and PPM.

\subsection{Variances of group and individual contributions}

Since lower marginal penalties imply a weaker link between an individual's contribution and her eventual payoff, the reduced incentive to select a precise contribution level may also induce a greater variance in contributions. The upper panel of Figure 3 shows that, in fact, the contributions under UPC appear more widely distributed around the symmetric contribution level, compared to PPM and PR; Figure 2 also shows different mechanisms lead to different variances in group contributions across sessions. A subject-period mixed effects model of the individual contributions reveals UPA and UPC have significantly higher variance in the homogeneous environment $(p<0.001)$ than PR and PPM, which are not significantly different; they are significantly higher in the heterogeneous environment as well (UPC vs. all the others with $p<0.001$, UPA vs. PPM with $p=0.016$ ), but UPA is not significantly higher than PR $(p=0.605) .{ }^{15}$ Similarly, a group-period mixed effects model reveals UPA and UPC have significantly higher variance in the homogenous environment (vs. PPM and PR with $p<0.01$ ), but UPC's significance is borderline in the heterogeneous environment (vs. PPM with $p=0.063$ and vs. PR with $p=0.115)$. This suggests the particular power of the uniform price mechanisms in encouraging higher contributions in the face of uncertainty about others' contributions, as we observe higher contributions despite increased uncertainty.

\footnotetext{
${ }^{14}$ Across the values associated with contributions within one standard deviation of the average UPC uniform price (4.66-8.28) UPC generates contributions averaging 0.567 of induced value, significantly higher than PPM (0.491, $p=0.037$ by rank sum test); UPC is insignificantly higher than $\mathrm{PR}(0.519, p=0.506)$.

${ }^{15}$ Following Marks and Croson (1998), we use two-factor (individual-period or group-period specific) random effects models to test for differences the variance of individual and total group contributions in the homogeneous and heterogeneous environments. The dependent variable is calculated as the squared distance from each individual's (group's) contribution to the average individual (group) contribution for each mechanism treatment in each period. The independent variables are the mechanism dummies using PPM as the baseline. Table 4 in the appendix shows the regression results.
} 


\section{Discussion}

This paper introduces two new mechanisms for threshold public goods, based on uniform price auctions: the uniform price auction mechanism (UPA) and the uniform price cap mechanism (UPC). It seeks to establish whether they perform better than the widely studied provision point mechanism without a rebate (PPM), and with a contribution-proportional rebate (PR). We first characterize these four mechanisms using the concepts of undominated perfect equilibrium (UPE) and the marginal payment penalty associated with over-contribution. We test the relative performance predictions through experiments characterized by complete information, with both homogeneous and heterogeneous values, and random regrouping between periods. Overall, the novel mechanisms improve upon those in the literature: UPA generated higher contributions, and UPC generated higher contributions and provision rates.

Understanding how the uniform price mechanisms generate higher contributions necessitates enhancing the equilibrium prediction provided by UPE: UPE establishes a continuum of equilibria for each mechanism, but coordinating on one among the equally refined equilibria causes groups to consistently make aggregate contributions that differ from the provision point, but with deviations that vary systematically by mechanism. It is in facilitating this coordination — off the equilibrium path - that differences among these mechanisms arise, and the differences that drive behavior are explained by the expected marginal penalty. The uniform price mechanisms UPA and UPC, both involving contribution ranges with zero marginal penalty, generate significantly higher group contributions than PR, which has a marginal penalty between -1 and 0 , which in turn attracts contributions higher than PPM, which has a marginal penalty of -1. These group contributions lead to a parallel order of provision rate, except for UPA which has more restrictive provision rules.

Examining the revealed strategy profiles, we find the coordination problem persists because many individual subjects are constantly adjusting their contributions in an effort to minimize their share of the cost when the good is provided. Provision, then, depends on some subjects offering above their equal share. The role of the rebate structure is to reduce the cost of making a high offer, so it may meet other high offers for purposes of coordination on disadvantageous asymmetric equilibria, making the mechanism more robust to cheap riding. Understanding this may be helpful in designing additional novel mechanisms, and identifying rebate structures best 
suited to specific applications with different information structures, distributions of values, and ratios between provision point and aggregate value.

That marginal penalty operates through facilitating coordination is supported by the observation that differences among mechanisms become more pronounced in environments where coordination is more difficult. In our experiment, the heterogeneous value treatment complicates coordination, since individual subjects may adopt different notions of "fair share" based on nominal contribution or share of value contributed. This also explains the mixed results comparing PPM and PR: Marks and Croson (1998) find no difference because they observe the same group of five homogeneous subjects playing the same game over 25 periods under complete information, a relatively easy coordination problem; while Gailmard and Palfrey (2005) find a significant difference because they reshuffle group members playing a Bayesian game. Our environment is of intermediate complexity - two homogeneous groups of five people reshuffled among 10 in each period with complete information-and we have results consistent with Gailmard and Palfrey, suggesting Marks and Croson's environment did not present a sufficient coordination challenge to fully evaluate their marginal penalty argument.

This focus on facilitating coordination may also explain other stylized facts about behavior in public goods games. For example, PR becomes more effective in larger groups and pushes contributions toward full value (demand) revelation (e.g., Rondeau et al., 1999; Spencer et al., 2009) in one-shot Bayesian games. Uncertainty over the total contributions of others may increase with group size, especially with private value information, and thus a rebate that reduces the risk associated with larger offers induces much higher contributions. Current work is extending the framework presented here to games of incomplete information, where we conjecture that not knowing where one falls in the realized value distribution yet further complicates coordination. The model integrates over the distribution of others' values in calculating the expected marginal penalty to arrive at a Bayesian Nash equilibrium prediction. Empirically, however, this additional uncertainty could introduce enough noise into contributions that subjects may have a hard time establishing an expectation of the marginal penalty associated with different contribution levels, obscuring differences among mechanisms.

The novel uniform price UPA and UPC mechanisms do improve upon existing budgetbalancing mechanisms in value revelation and provision of a threshold public good. If one is valuing a non-market good — whose eventual provision will be by an efficient coercive tax- 
through a small-scale pilot with a real money, real public good to reduce hypothetical bias, then UPA minimizes the bias of welfare estimates caused by cheap riding (cf. Swallow et al, 2008). If efficient provision of the public good is the major concern, UPC represents a meaningful improvement over the well studied PPM and PR. This is especially true if the group being targeted for provision has a relatively large proportion of high value people, in which case the zero or low marginal penalty in the uniform price mechanisms allows them to make larger contributions, more fully revealing their values and increasing prospects for provision.

\section{Acknowledgements}

The authors would like to thank the Editor and three anonymous reviewers for insightful comments and suggestions that have improved the paper. This work was supported under USDA/NIFA Award No. 2009-55401-05038.

\section{References}

Alboth, D., Lerner, A. and Shalev, J. (2001), Profit Maximizing in Auctions of Public Goods, Journalof Public Economic Theory, 3 (4): 501-525.

Bagnoli, M. and Lipman, B. L. (1989), Provision of Public Goods: Fully Implementing the Core through Private Contributions, Review of Economic Studies, 56 (4): 583-601.

Bagnoli, M. and McKee, M. (1991), Voluntary Contribution Games: Efficient Private Provision of Public Goods, Economic Inquiry, 29 (2): 351-366.

Bagnoli, M., Bendavid, S. and McKee, M. (1992), Voluntary Provision of Public Goods: the Multiple Unit Case, Journal of Public Economics, 47 (1): 85-106.

Barbieri, S. and Malueg, D. A. (2008), Private Provision of a Discrete Public Good: Efficient Equilibria in the Private-Information Contribution Game, Economic Theory, 37 (1): 51-80.

Barbieri, S. and Malueg, D. A. (2010a), Threshold Uncertainty in the Private-Information Subscription Game, Journal of Public Economics, 94 (11-12): 848-861.

Barbieri, S. and Malueg, D. A. (2010b), Profit-Maximizing Sale of a Discrete Public Good via the Subscription Game in Private-Information Environments, B E Journal of Theoretical Economics, 10 (1): Article 5.

Bush, G., Hanley, N., Moro, M., and Rondeau, D. (2013), Measuring the Local Costs of Conservation: A Provision Point Mechanism for Eliciting Willingness to Accept 
Compensation, Land Economics, 89 (3):490-513.

Cason, T. N. and Gangadharan, L. (2004), Auction Design for Voluntary Conservation Programs, American Journal of Agricultural Economics, 86 (5): 1211-1217.

Cason, T. N. and Gangadharan, L. (2005), A Laboratory Comparison of Uniform and Discriminative Price Auctions for Reducing Non-Point Source Pollution, Land Economics, 81 (1): 51-70.

Champ, P. A., Flores, N. E., Brown, T. C. and Chivers, J. (2002), Contingent Valuation and Incentives, Land Economics, 78 (4): 591-604.

Chen, Y. (2008), Incentive-Compatible Mechanisms for Pure Public Goods: A Survey of Experimental Research. In: Charles, R.P. and. Smith, V.L., Editors, 2008. Handbook of Experimental Economics Results. North-Holland, Amsterdam, The Netherlands, Volume 1: 625-643.

Davis, D. and Holt, C. (1993), Experimental Economics, Princeton University Press, Princeton, NJ, p.317 - 379 .

Evans, M. F., Vossler, C. A. and Flores, N. E. (2009), Hybrid Allocation Mechanisms for Publicly Provided Goods, Journal of Public Economics, 93 (1-2): 311-325.

Fischbacher, U. (2007), z-Tree: Zurich Toolbox for Ready-made Economic Experiments, Experimental Economics, 10 (2): 171-178.

Gailmard, S. and Palfrey, T. R. (2005), An Experimental Comparison of Collective Choice Procedures for Excludable Public Goods, Journal of Public Economics, 89 (8): 1361-1398.

Haskell, J., Uchida, E., Swallow, S.K., and Uchida, H. (2010), Willingness-to-Pay for Ecosystem Services in Rhode Island: Do Payment Elicitation Mechanisms Matter? University of Rhode Island working paper, Retrieved (2016.08.19) from http://www.webmeets.com/WCERE/2010/prog/viewpaper.asp?pid=1037

Isaac, R. M., Schmidtz, D. and Walker, J. M. (1989), The Assurance Problem in a Laboratory Market, Public Choice, 62 (3): 217-236.

Jack, B. K., Leimona, B. and Ferraro, P. J. (2009), A Revealed Preference Approach to Estimating Supply Curves for Ecosystem Services: Use of Auctions to Set Payments for Soil Erosion Control in Indonesia, Conservation Biology, 23 (2): 359-367.

Laussel, D. and Palfrey, T. R. (2003), Efficient Equilibria in the Voluntary Contributions Mechanism with Private Information, Journal of Public Economic Theory, 5 (3): 449-478. 
Ledyard, J.O., (1995). Public Goods: A Survey of Experimental Research. In: Kagel, J.H. and Roth, A.E., Editors, 1995. The Handbook of Experimental Economics, Princeton University Press, Princeton, New Jersey, pp. 111-194.

Marks, M. and Croson, R. (1998), Alternative Rebate Rules in the Provision of A Threshold Public Good: An Experimental Investigation, Journal of Public Economics, 67 (2): 195-220.

McBride, M. (2006), Discrete Public Goods under Threshold Uncertainty, Journal of Public Economics, 90 (6-7): 1181-1199.

Menezes, F. M., Monteiro, P. K. and Temimi, A. (2001), Private Provision of Discrete Public Goods with Incomplete Information, Journal of Mathematical Economics, 35 (4): 493-514.

Nitzan, S. and Romano, R. E. (1990), Private Provision of A Discrete Public Good with Uncertain Cost, Journal of Public Economics, 42 (3): 357-370.

Rondeau, D., Poe, G. L. and Schulze, W. D. (2005), VCM or PPM? A Comparison of the Performance of Two Voluntary Public Goods Mechanisms, Journal of Public Economics, 89 (8): 1581-1592.

Rondeau, D., Schulze, W. D. and Poe, G. L. (1999), Voluntary Revelation of the Demand for Public Goods Using A Provision Point Mechanism, Journal of Public Economics, 72 (3): 455-470.

Spencer, M. A., Swallow, S. K., Shogren, J. F. and List, J. A. (2009), Rebate Rules in Threshold Public Good Provision, Journal of Public Economics, 93 (5-6): 798-806.

Suleiman, R. and Rapoport, A. (1992), Provision of Step-Level Public Goods with Continuous Contribution, Journal of Behavioral Decision Making, 5 (2): 133-153.

Swallow, S. K. (2013), Demand-Side Value for Ecosystem Services and Implications for Innovative Markets: Experimental Perspectives on the Possibility of Private Markets for Public Goods, Agricultural and Resource Economics Review, 42 (1): 33-56.

Swallow, S. K., Smith, E. C., Uchida, E., and Anderson, C. M. (2008), Ecosystem Services beyond Valuation, Regulation, and Philanthropy: Integrating Consumer Values into the Economy. Choices, 23(2):47-52.

van Damme, E. (1983), Refinements of the Nash Equilibrium Concept, Springer-Verlag, Berlin. 